\title{
Aqueous electrolytes confined within functionalized silica nanopores
}

\author{
Pablo E. Videla, ${ }^{1}$ Jonàs Sala, ${ }^{2}$ Jordi Martí, ${ }^{2}$ Elvira Guàrdia, ${ }^{2}$ and Daniel Laria ${ }^{1,3, a)}$ \\ ${ }^{1}$ Departamento de Química Inorgánica Analítica y Química-Física e INQUIMAE, \\ Facultad de Ciencias Exactas y Naturales, Universidad de Buenos Aires, Ciudad Universitaria, \\ Pabellón II, 1428 Buenos Aires, Argentina \\ ${ }^{2}$ Departament de Física i Enginyeria Nuclear, Universitat Politècnica de Catalunya-Barcelona Tech, \\ B4-B5 Campus Nord, 08034 Barcelona, Spain \\ ${ }^{3}$ Departamento de Física de la Materia Condensada, Comisión Nacional de Energía Atómica, \\ Avenida Libertador 8250, 1429 Buenos Aires, Argentina
}

(Received 7 June 2011; accepted 11 August 2011; published online 9 September 2011)

\begin{abstract}
Molecular dynamics simulations have been carried out to investigate structural and dynamical characteristics of $\mathrm{NaCl}$ aqueous solutions confined within silica nanopores in contact with a "bulk-like" reservoir. Two types of pores, with diameters intermediate between $20 \AA$ and $37.5 \AA$, were investigated: The first one corresponded to hydrophobic cavities, in which the prevailing wall-solution interactions were of the Lennard-Jones type. In addition, we also examined the behavior of solutions trapped within hydrophilic cavities, in which a set of unsaturated O-sites at the wall were transformed in polar silanol $\mathrm{Si}-\mathrm{OH}$ groups. In all cases, the overall concentrations of the trapped electrolytes exhibited important reductions that, in the case of the narrowest pores, attained 50\% of the bulk value. Local concentrations within the pores also showed important fluctuations. In hydrophobic cavities, the close vicinity of the pore wall was coated exclusively by the solvent, whereas in hydrophilic pores, selective adsorption of $\mathrm{Na}^{+}$ions was also observed. Mass and charge transport were also investigated. Individual diffusion coefficients did not present large modifications from what is perceived in the bulk; contrasting, the electrical conductivity exhibited important reductions. The qualitative differences are rationalized in terms of simple geometrical considerations. (C) 2011 American Institute of Physics. [doi:10.1063/1.3632050]
\end{abstract}

\section{INTRODUCTION}

In recent years, a large body of research work has been focused on the analysis of aqueous electrolytes trapped within cylindrical-like pores with dimensions in the nanometer range. Two main reasons have spurred the interest in these liquid phases. From a biological perspective, confined salt-solutions represent appropriate benchmarks to analyze mass and charge transport through complex biological membranes and ionic channels. ${ }^{1-5}$ In addition, the subject is relevant in many processes with practical applications in the area of nanofluidics, ${ }^{6,7}$ such as selective fluxes through membranes controlled by size, ${ }^{8}$ wall-functionalization ${ }^{9,10}$ or external fields, ${ }^{11-13}$ desalinization processes, ${ }^{14}$ nanopipets, ${ }^{15}$ and osmotic-driven fluxes, ${ }^{16,17}$ to cite a few relevant examples. In all these processes, the adequate microscopic characterization of the dynamics of the trapped liquids represents a key element for the correct interpretation of their performances.

By now, it is well established that the behavior of liquid phases under nanometric confinement comes as a result of a complex interplay between inter- and intra-molecular interactions, conjugated with the particular geometrical restrictions of the environments and the effective interactions prevailing at the domain walls. ${ }^{18}$ These effects may give rise to collective responses of the fluids - such as segregation phenomena, ${ }^{19-21}$ modifications in phase diagrams, ${ }^{2-28}$ and

\footnotetext{
a) Author to whom correspondence should be addressed. Electronic mail: dhlaria@cnea.gov.ar.
}

drying transitions ${ }^{29-33}$ - which are absent in more conventional, bulk phases. On the dynamical side, confinement effects are equally dramatic and are normally portrayed in terms of important retardations in the translational and rotational motions. $^{34-37}$

In what follows, we will present results from molecular dynamics experiments in which we examined the behavior of aqueous $\mathrm{NaCl}$ solutions confined within silica pores. There is a large amount of scientific production devoted to the analysis of these mixtures under nanoconfinement. Most notably are those oriented to the analysis of fluids within model hydrophobic nanotubes ${ }^{38-44}$ and in slit-shaped silica and carbon nanopores. ${ }^{45-49}$ Our motivation in performing this new set of simulations was to analyze pore size and wallfunctionalization effects on the equilibrium solvation structures and the dynamics of the solute species within the pores. Special care was taken to model the confined liquid phase under realistic conditions, i.e., in contact with a bulk solution. In this way we could gauge the modifications operated in the global and local density fields, taking as a reference what is perceived at the bulk. Interestingly, this structural description provided physically sound arguments to rationalize the qualitative differences observed in the dynamical coefficients describing mass and charge transport through the pores.

The organization of the paper is as follows: Details of the model and simulation procedure are presented in Sec. II. The main results are discussed in Sec. III. Our concluding remarks appear in Sec. IV. 


\section{MODEL AND SIMULATION PROCEDURE}

The molecular dynamics experiments were performed on fully periodic model systems, mimicking a cylindrical pore (I) of length $\ell$, with a reservoir (II and III) filled with $\mathrm{NaCl}$ aqueous solutions, at a global salt concentration close to $c \sim 1 \mathrm{M}$ (see Fig. 1). The cylindrical pore was aligned with its axis parallel to the $z$-direction and occupied the central, $|z| \leq 16.5 \AA$, region of the simulation box. The lengths of the simulation box were set to $L_{x}=L_{y}=51 \AA$ and $L_{z} \sim 65 \AA$.

The silica pores were generated following a procedure described in full detail in previous papers ${ }^{20,35,51-53}$ so, for the sake of conciseness we will only present a brief description of the main steps. The starting point was the consideration of a sample of fused $\mathrm{SiO}_{2}$, at a global density of $2.2 \mathrm{~g} \mathrm{~cm}^{-3}$, contained within a simulation box with lengths $L_{x}=L_{y}=51 \AA$ and $L_{z}=33 \AA$, at temperatures close to $T \sim 8000 \mathrm{~K}$. After immobilizing a central cylindrical section of radius $R$ of the sample along the $z$ axis, the surrounding portion was annealed down to $T \sim 300 \mathrm{~K}$. The central resist was then removed, leaving a cylindrical pore across a solid silica slab. To gauge confinement effects, three different values of $R=10,15$, and $17.5 \AA$ were examined. In the latter case, the lengths of the simulation box were set to $L_{x}=L_{y}=55 \AA$ and $L_{z} \sim 89 \AA$.

In addition, we also examined effects arising from modifications in the effective wall-solution interactions. To that end, we considered two types of pores: (i) the first class - hereafter referred to as hydrophobic cavities (HOCs) corresponds to pores in which wall-solution interactions were exclusively of the Lennard-Jones type; (ii) in addition, we examined electrolyte confinement within hydrophilic cavities (HICs), in which unsaturated O-sites at the interface were transformed into polar $\mathrm{Si}-\mathrm{OH}$ groups. In accordance with experimental information, ${ }^{50}$ the surface density of silanol groups was set to $3 \mathrm{~nm}^{-2}$. The final stage of the initial equilibration procedure involved bringing together the solid sample with a $\mathrm{NaCl}$ aqueous solution, which was allowed to

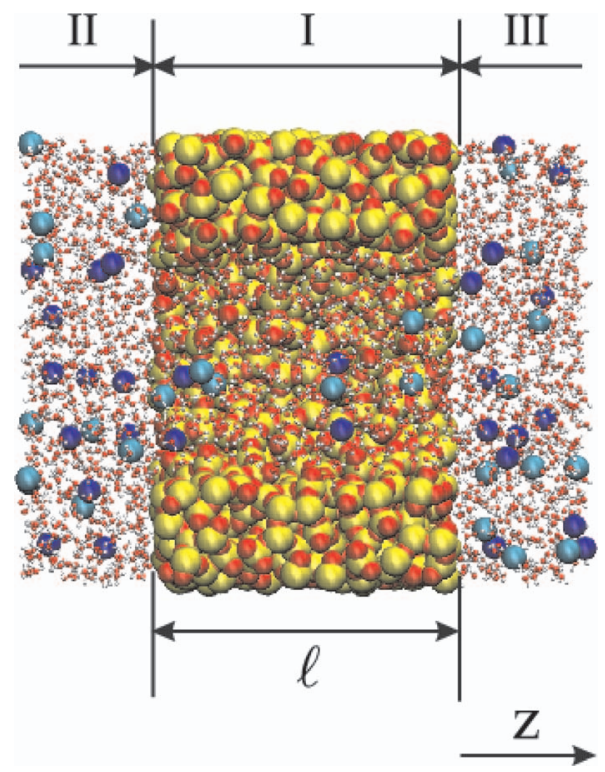

FIG. 1. Snapshot of a cut of the simulation box with the hydrophobic silica pore in the middle region.
TABLE I. Potential parameters.

\begin{tabular}{lccr}
\hline \hline Site & $\begin{array}{c}\epsilon \\
(\mathrm{kcal} / \mathrm{mol})\end{array}$ & $\begin{array}{c}\sigma \\
(\AA)\end{array}$ & \multicolumn{1}{c}{$\begin{array}{c}\mathrm{q} \\
(e)\end{array}$} \\
\hline a. Water & & & \\
O & 0.152 & 3.15 & -0.834 \\
H & & & 0.417 \\
b. Ions & & & \\
Na & 0.1 & 2.584 & 1.0 \\
Cl & 0.1 & 4.401 & -1.0 \\
c. Silica & & & \\
O (HOC) & 0.155 & 3.154 & \\
Si (HOC) & 0.128 & 3.795 & -0.71 \\
O (silanol) & 0.155 & 3.154 & 0.31 \\
Si (silanol) & 0.128 & 3.795 & 0.40 \\
H (silanol) & & & \\
\hline \hline
\end{tabular}

permeate through the pore. To facilitate the filling, the temperature was taken up to $473 \mathrm{~K}$ during 2 ns. From then on, the liquid phase was allowed to re-equilibrate at ambient conditions for about $5 \mathrm{~ns}$, until the global density of the electrolytes within the pore remained fairly constant. During this period, the value of $L_{z}$ was gradually adjusted so as to bring the water local density at the $z \sim L_{z} / 2$, bulk region in agreement with the experimental value of a $c=1 \mathrm{M}, \mathrm{NaCl}$ aqueous solution, i.e., $\rho_{w}^{\text {bulk }}=3.27 \times 10^{-2} \AA^{-3}$. With this procedure, the resulting salt concentrations in the bulk regions were intermediate between $c_{\mathrm{blk}}=1.05 \mathrm{M}$ and $1.2 \mathrm{M}$, depending on the particular pore size and the wall-solution interaction considered. In simulations involving the smaller pores, the liquid phases contained typically $N_{w} \sim 3500$ water molecules and $N_{\mathrm{Na}^{+}}=N_{\mathrm{Cl}^{-}} \sim 65$ ions and twice as much, in simulations with pores with radius $R=17.5 \AA$.

The trajectories were generated using the NAMD package $^{54}$ and corresponded to the microcanonical ensemble. The potential energy of the system was considered to be pairdecomposable, with site-site interactions involving LennardJones and Coulomb contributions. For the water molecules, we adopted the TIP3P model (see Table Ia), ${ }^{55}$ whereas for the electrolytes, the Lennard-Jones energy and length parameters and partial charges were taken from Ref. 30 and are listed in Table Ib. Parameters for interactions involving sites in the silica pore were taken from Ref. 56 and appear in Table Ic. Usual arithmetic and geometrical means were implemented to describe cross interactions. To handle the long range nature of the Coulomb interactions we implemented a particle mesh procedure. ${ }^{57}$ Statistics of the structural characteristics of the confined solutions were collected from 5 statistically independent runs, each one lasting $10 \mathrm{~ns}$.

\section{RESULTS}

\section{A. Equilibrium results}

The first aspect that we will examine deals with the overall modifications of the global concentrations within the pore, compared to the ones prevailing in the bulk regions. The 
analysis of normalized local density fields of the type

$$
\rho_{\alpha}^{\mathrm{cyl}}(z)=\frac{1}{\pi R^{2}} \sum_{i}\left\langle\delta\left(z-z_{i}^{\alpha}\right)\right\rangle_{R}
$$

provides a simple route to monitor these differences. In the previous equation, $\left(x_{i}^{\alpha}, y_{i}^{\alpha}, z_{i}^{\alpha}\right)$ represent the coordinates of the $i$ th particle of species $\alpha$, measured from a coordinate system centered at the center of mass of the pore and $\langle\cdots\rangle_{R}$ denotes an average restricted to those molecules whose distance to the $z$-axis was less than $R$.

Results of $\rho_{\alpha}^{\text {cyl }}(z)\left(\alpha=\mathrm{H}_{2} \mathrm{O}, \mathrm{Na}^{+}, \mathrm{Cl}^{-}\right)$normalized by the corresponding bulk values in HOCs and HICs are presented in Figs. 2 and 3, respectively. At a first glance, one observes that, in both pores, confinement effects lead to important reductions of the overall electrolyte concentrations. As expected, these effects become more vivid in narrower pores. For example, within HOCs, as we move from $R$ $=17.5 \AA$ to $R=10 \AA$, the global concentrations within the pores drop from $\sim 0.8 \rho_{\alpha}^{\text {blk }}$ down to $\sim 0.5 \rho_{\alpha}^{\text {blk }}$. These reductions correspond to free energy barriers for the electrolyte confinement of the order of $\Delta F=-k_{B} T \ln \left[\rho_{\alpha}^{\text {cyl }}(z\right.$ $\left.\sim 0) / \rho_{\alpha}^{\mathrm{blk}}\right] \sim 0.1-0.4 \mathrm{kcal} \mathrm{mol}^{-1}$. We remark that $R \sim 10$ $\AA$ represents a threshold value below which hydrophobic pores exhibit spontaneous drying transitions; in fact, we verified that narrower pores (with radii, say, down to $9 \AA$ ), filled with the solution at $T \sim 473 \mathrm{~K}$, become empty, as the temperature is lowered at ambient conditions, on a timescale of a few tens of picoseconds. This phenomenon is well documented and is the result of a delicate interplay between geometrical characteristics of the confined region, the overall

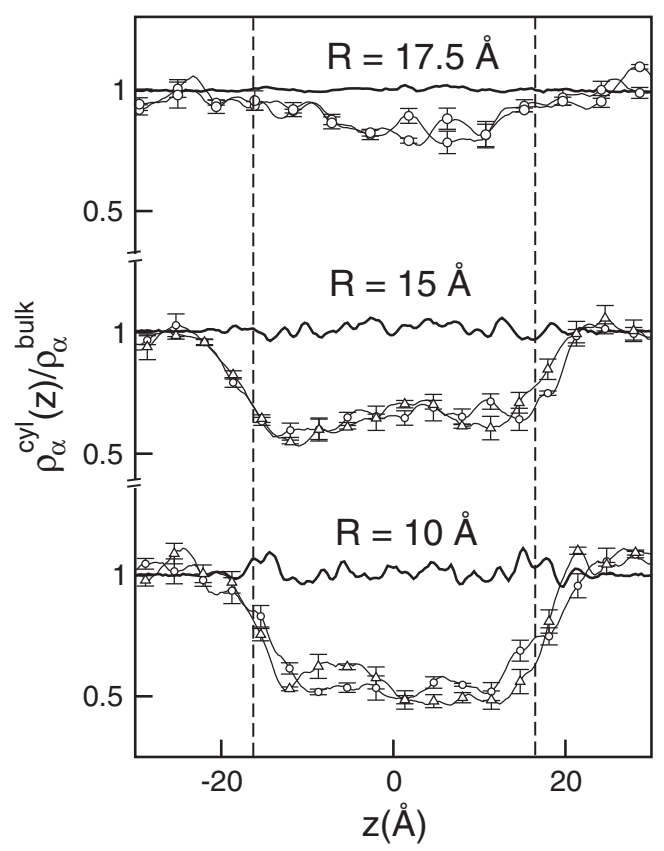

FIG. 2. Local density fields for water and ionic species along the $z$ axis for hydrophobic pores with different radii. Sampling within a cylindrical volume of radius $R$ are shown for water oxygens (solid line), $\mathrm{Na}^{+}$(circles), and $\mathrm{Cl}^{-}$ (triangles). The vertical dashed lines denote the pore boundaries. The error bars correspond to standard deviations from five statistically independent trajectories (see text).

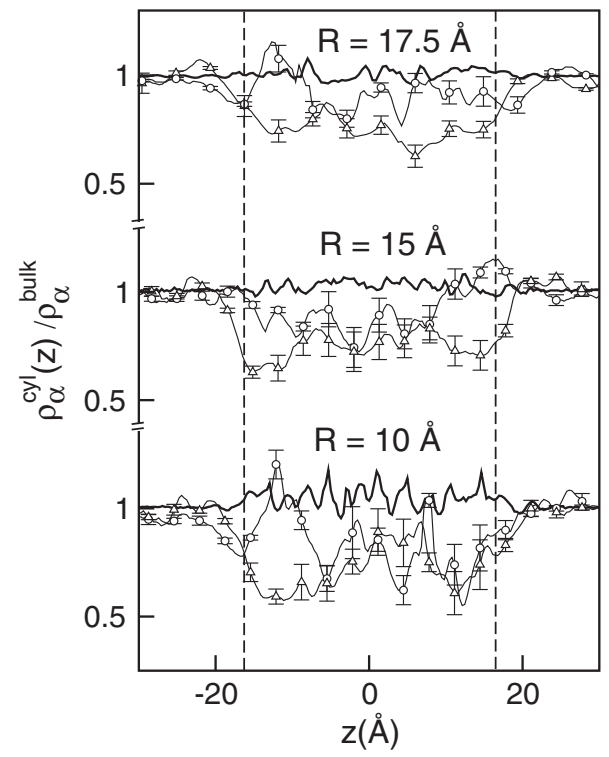

FIG. 3. Same as Fig. 2 for hydrophilic pores.

characteristics of the prevailing interactions, and thermodynamic conditions. ${ }^{31}$

Coming back to the joint analysis of the plots displayed in Figs. 2 and 3, effects from wall functionalization upon the overall electrolyte concentration within the pores get translated into more irregular profiles for the HIC cases. These more prominent fluctuations not only can be observed in the individual ion profiles but they are also evident in the solvent ones. Moreover, modifications in the $\mathrm{Na}^{+}$plot appear somewhat more marked than those observed for $\mathrm{Cl}^{-}$. As we will see shortly, these characteristics respond to the equally irregular arrangement of the polar silanol groups at the pore wall.

Within the pores, inhomogeneities in the density fields also manifest along the radial direction. In Fig. 4 we

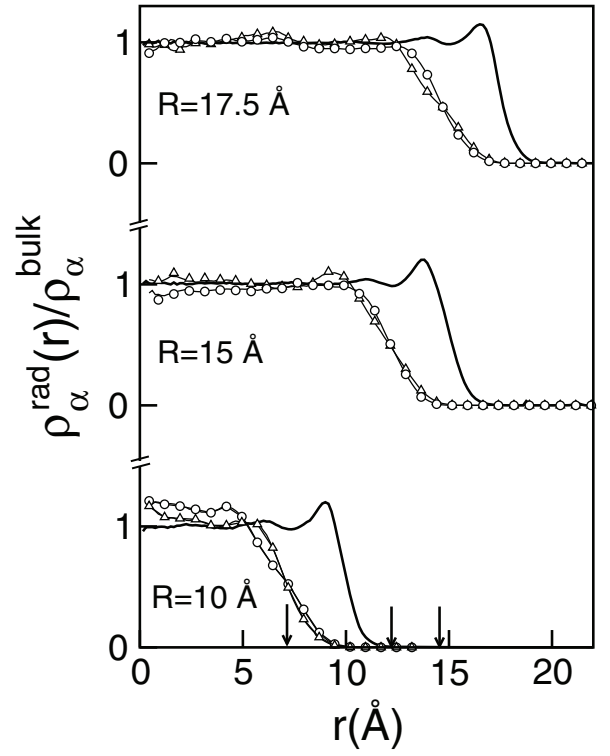

FIG. 4. Density fields within the hydrophobic pore along the radial direction. Same labeling as Fig. 2. The arrows in the $x$ axis indicate the values of $\widetilde{R}$ (see text). 


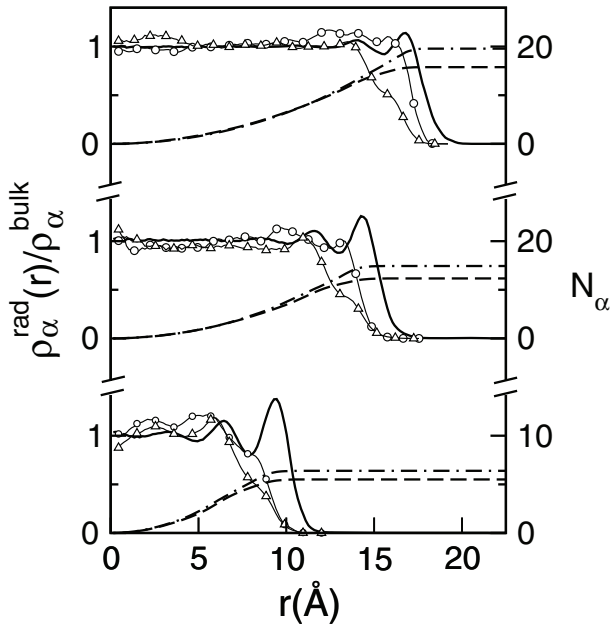

FIG. 5. Left axis: Density fields within hydrophilic pores along the radial direction. Same labeling as Fig. 4. Right axis: Electrolyte cumulative integrals $N_{\alpha} \cdot\left(\mathrm{Na}^{+}\right.$: dashed-dotted lines, $\mathrm{Cl}^{-}$: dashed lines).

present normalized local density fields along this direction in hydrophobic cavities, namely:

$$
\rho_{\alpha}^{\mathrm{rad}}(r)=\frac{1}{2 \pi \ell r} \sum_{i}\left\langle\delta\left(r-r_{i}^{\alpha}\right)\right\rangle_{\mathrm{pore}},
$$

where $r_{i}^{\alpha}$ denotes the distance from the $i$ th molecule of species $\alpha$ to the pore axis and $\langle\cdots\rangle_{\text {pore }}$ denotes an average taken from molecules lying within the pore, i.e., sites whose coordinates satisfy $\left|z_{i}^{\alpha}\right| \leq \ell / 2$. The plots show that, regardless of the pore size, the walls are coated exclusively by water. Note that the thickness of this surface layer, $\Delta$, is of similar order to the diameter of a water molecule, $\sim 2.5-3 \AA$. We remark that these observations are in agreement with results reported for aqueous electrolytes within carbon nanotubes. ${ }^{38}$ As we move to inner water layers, the concentrations quickly attain their bulk values in the three pores, although in the narrowest one, the ion populations at the center slightly surpass that value.

Plots of $\rho_{\alpha}^{\mathrm{rad}}(r)$ in HICs are displayed in Fig. 5 and differ from those described in the previous paragraphs: (i) First, the water profiles exhibit increments in their fluctuations, specially in the narrowest, $R=10 \AA$ pore case. (ii) Second, in the outer layer in contact with the pore walls, one observes local increments of the $\mathrm{Na}^{+}$concentrations. The analysis of the cumulative integrals of the ionic densities, $N_{\alpha}(r)$, is instructive. Note that, for the three pore sizes, the two curves detach from each other beyond a radial distance which practically coincides with the location of the outer solvent shell. Expressed in terms of the global salt content within the pores, these increments roughly represent $\sim 25 \%$ of the global $\mathrm{Na}^{+}$ concentrations. Incidentally, we remark that the prevalence of $\mathrm{Na}^{+}$wall solvation states - which has also been reported ${ }^{47}$ in silica pores with slit geometry - responds mainly to steric effects, since the smaller size of the cations facilitates their stabilization within irregular wall domains surrounding some silanol groups. In this respect we found no correlations between the local fluctuations in the three profiles of $\rho_{\mathrm{Na}}^{\text {cyl }}(z)$ and the overall density of silanol groups along the $z$-direction, so we tend to believe that the characteristics of the resulting ion density profiles reflect the availability of binding sites at the vicinity of a few selected $\mathrm{Si}-\mathrm{O}-\mathrm{H}$ groups; (iii) Similar to what we found in hydrophobic pores, as we move to inner solvent shells, both local concentrations become comparable and similar in magnitude to the one found in the bulk (regions II and III in Fig. 1 ).

\section{B. Dynamical analysis}

We will now move to the dynamical analysis that will be focused on time correlation functions describing diffusive motions and the electrical conductivity along the axial direction. Individual diffusion coefficients were obtained from the limiting slopes of $\mathcal{R}_{\alpha}^{2}$, the root mean square displacements along the axial directions:

$$
D_{\alpha}^{\|}=\lim _{t \rightarrow \infty} \frac{\left\langle\left|z_{i}^{\alpha}(t)-z_{i}^{\alpha}(0)\right|^{2}\right\rangle}{2 t} ;
$$

whereas estimates for the conductivity were computed from time integrals of the current-current time correlation function, namely:

$$
\begin{aligned}
\sigma & =\frac{1}{k_{B} T V} \int_{0}^{\infty}\left\langle J^{\|}(t) \cdot J^{\|}(0)\right\rangle \mathrm{d} t ; \\
J^{\|}(t) & =\sum_{\alpha} \sum_{i} q_{\alpha} \dot{z}_{i}^{\alpha} .
\end{aligned}
$$

In the previous equations, $V=\pi R^{2} \ell$ denotes the pore volume and $q_{\alpha}$ is the charge of species $\alpha$. All correlation functions were computed by restricting the sampling to contributions from those ions that were present within the pore at times 0 and $t$.

Results for mass and charge transport coefficients in HOCs are listed in columns 2-4 of Table IIa. Surprisingly, when compared to bulk solution results, the ionic diffusion coefficients only present a $\sim 10 \%$ drop in the narrowest pores while they are practically the same, for wider ones. Moreover, this constancy clashes with the results observed in the diffusion of simple polar solvents, where diffusion coefficients are

\begin{tabular}{|c|c|c|c|c|c|}
\hline $\begin{array}{l}R \\
(\AA)\end{array}$ & $\begin{array}{c}D_{\mathrm{Na}^{+}}^{\|} \\
\left(10^{-5} \mathrm{~cm}^{2} \mathrm{~s}^{-1}\right)\end{array}$ & $\begin{array}{c}D_{\mathrm{Cl}^{-}}^{\|} \\
\left(10^{-5} \mathrm{~cm}^{2} \mathrm{~s}^{-1}\right)\end{array}$ & $\begin{array}{c}\sigma \\
\left(\Omega^{-1} \mathrm{~m}^{-1}\right)\end{array}$ & $\begin{array}{l}\widetilde{R}^{\mathrm{a}} \\
(\AA)\end{array}$ & $\begin{array}{c}\tilde{\sigma} \\
\left(\Omega^{-1} \mathrm{~m}^{-1}\right)\end{array}$ \\
\hline \multicolumn{6}{|c|}{ a. Hydrophobic pores } \\
\hline 10 & $0.9 \pm 0.1$ & $1.2 \pm 0.1$ & $4.4 \pm 0.1$ & 7.5 & 7.8 \\
\hline 15 & $1.1 \pm 0.1$ & $1.3 \pm 0.1$ & $5.0 \pm 0.1$ & 12.5 & 7.2 \\
\hline 17.5 & $1.1 \pm 0.1$ & $1.3 \pm 0.1$ & $5.4 \pm 0.2$ & 15.0 & 7.4 \\
\hline \multicolumn{6}{|c|}{ b. Hydrophilic pores } \\
\hline 10 & $0.6 \pm 0.1$ & $0.8 \pm 0.2$ & $4.5 \pm 0.2$ & 7.6 & 7.8 \\
\hline 15 & $0.8 \pm 0.2$ & $1.1 \pm 0.2$ & $5.5 \pm 0.4$ & 13.0 & 7.3 \\
\hline 17.5 & $0.8 \pm 0.2$ & $1.1 \pm 0.2$ & $6.3 \pm 0.4$ & 15.5 & 8.0 \\
\hline Bulk $^{b}$ & 1.1 & 1.3 & 7.8 & & \\
\hline
\end{tabular}
known to exhibit much more drastic reductions. ${ }^{34-36}$ We also remark that, in all cases investigated, we found no evidence of

TABLE II. Transport coefficients for confined aqueous electrolytes.

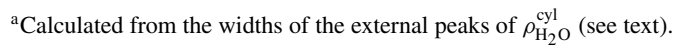

${ }^{\mathrm{b}}$ Reference values for a $c=1 \mathrm{M}$, bulk solution taken from Ref. 56. 
sublinear behaviors in the temporal dependences of any of the $\mathcal{R}_{\alpha}^{2}(t)$ investigated, a fact that would reveal modifications in the bulk-like mechanisms controlling electrolyte mass transport. On the other hand, the electric conductivity does show more marked modifications: note that $\sigma$ falls from $0.7 \sigma^{\text {bulk }}$ for $R=17.5 \AA$ pores down to $0.55 \sigma^{\text {bulk }}$ for $R=10 \AA$ ones. The analysis of transport coefficients in HICs listed in Table IIb suggests a similar scenario, although the retardations are somewhat more marked (see, for example, the case of $\mathrm{Na}^{+}$in the narrowest pore where the diffusion coefficient drops practically to half the bulk value).

Looking for clues to rationalize the qualitative differences between the behaviors of the two transport coefficients, we re-examined the structural description of the confined electrolytes and focused on the fact that, in both types of pores, the spatial confinements of the charged species were actually somewhat tighter than the ones estimated by the sole consideration of the dimensions of the pore. The characteristics of $\rho_{\alpha}^{\mathrm{rad}}(r)$ led us first to define the effective radii, $\widetilde{R}=R-\Delta$, taking into account the positions of the outer local minima in the $\rho_{\mathrm{O}}^{\mathrm{rad}}(r)$ profiles. We then evaluated effective conductivities $\widetilde{\sigma}$, by simply rescaling the original estimates of the pore volumes by the geometrical factors $(\widetilde{R} / R)^{2}$. Values of $\widetilde{R}$ and results for $\widetilde{\sigma}$ are shown in columns 5 and 6 of Table II. One can observe that practically all effective conductivities look similar in magnitude and much more comparable to the bulk results. Moreover, a similar argument could be invoked to explain the mild modifications of the ionic diffusion coefficients in HOCs, since the prevailing confinement would be concentrated in the central and dynamically fastest - region of the pore. In HICs, contributions from the tightly adsorbed $\mathrm{Na}^{+}$would bring down the diffusion coefficients. The general picture that emerges from these simple geometrical considerations suggests that the drop in the observed conductivity would simply reflect a reduction of the global electrolyte concentration, due to the presence of coating shells deprived of charged species in the HOC cases and tightly adsorbed $\mathrm{Na}^{+}$, with much minor contributions to the parallel current, in the hydrophilic pores.

\section{CONCLUDING REMARKS}

Our simulation results reveal important modifications in the global and local densities of aqueous electrolytes confined within silica pores in contact with bulk-like reservoirs. The latter is a key ingredient since it allows an adequate control of the local fluctuations of the individual concentrations within the confined domain, bringing the comparison between structural and dynamical characteristics of bulk and pore environments physically meaningful. Within pores with diameters of the order of $\sim 2-3 \mathrm{~nm}$, the global concentrations are intermediate between 0.5 and 0.8 times the ones observed in the bulk reservoir. From a local perspective, in hydrophobic cavities, the closest solvation shell of each ion is preserved, leading to an external coating layer in contact with the pore wall, composed exclusively of water. The presence of hydroxylated groups at the solid interface leads to the incorporation of excess $\mathrm{Na}^{+}$ions in the outer shell. This binding is controlled by the structural characteristics of the amorphous pore wall in the close vicinity of these polar groups, i.e., the availability of adsorption sites large enough to accommodate cationic species.

Mass transport coefficients in the pores exhibit only mild drops, which contrast with the more marked modifications observed in the ionic conductivity. Looking for clues to rationalize the observed qualitative differences between the behaviors of the two transport coefficients, we focused our attention on the characteristics of the spatial confinement. The simple consideration of a smaller, effective volume leads to a more unified description of $D_{\alpha}^{\|}$and $\tilde{\sigma}$ which would suggest that, in this pore-size regime, the mechanisms that control mass and charge transport would not differ from those prevailing in bulk in a sensible fashion.

Additional investigations to corroborate the range of validity of our conclusions are surely called for. We can think of two relevant questions in this respect: (i) First, one could speculate on possible modifications in the extent of the central segregation of the ionic species in cases involving bigger and more polarizable anions, such as $\mathrm{I}^{-}$, which are known to exhibit more propensity for surface solvation states, at least, in water/air interfaces, ${ }^{58}$ (ii) second, the particular structural characteristics of the pore walls is another issue that will require further examination. In order to clearly discern between effects from short-range and long-range Coulomb wallsolution interactions, we restricted our analysis to two model pores investigated that differed only in the addition of partial charges at a few selected $\mathrm{Si}-\mathrm{O}-\mathrm{H}$ groups at the interface. Further simulations focused on clarifying these two issues are currently carried out in our laboratory.

\section{ACKNOWLEDGMENTS}

D.L. is a staff member of CONICET (Argentina). J.S., E.G., and J.M. thank financial support from the "Direcció General de Recerca" of the Generalitat de Catalunya (Grant No. 2009-SGR-1003) and the "Ministerio de Ciencia e Innovación" (MICINN) of Spain (Grant No. FIS2009-13641C02-01). J.S. is a recipient of a FPI Spanish fellowship.

${ }^{1}$ C. F. Lopez, S. O. Nielsen, P. B. Moore, and M. L. Klein, Proc. Natl. Acad. Sci. U.S.A. 101, 4431 (2004).

${ }^{2}$ See, for example, Aquaporins, Current Topics in Membranes Vol. 51, edited by S. Hohmann, P. Agre, S. Nielsen, D. J. Benos, and S. A. Simon (Academic, San Diego, 2001).

${ }^{3}$ See, for example, B. Roux and M. Karplus, Annu. Rev. Biophys. Biomol. Struct. 23, 731 (1994).

${ }^{4}$ Z. Hu and J. Jiang, J. Membr. Sci. 324, 192 (2008).

${ }^{5}$ P. S. Crozier, R. L. Rowley, N. B. Holladay, D. Henderson, and D. D. Busath, Phys. Rev. Lett. 86, 2467 (2001).

${ }^{6}$ R. N. Schoch, J. Y. Han, and P. Renadu, Rev. Mod. Phys. 80, 839 (2008).

${ }^{7}$ A. Noy, H. G. Park, F. Fornasiero, J. K. Holt, C. P. Griogoropoulos, and O. Bakajin, Nanotoday 2, 22 (2007).

${ }^{8}$ K. B. Jirage, J. C. Hulteen, and C. R. Martin, Science 278, 655 (1997).

${ }^{9}$ B. J. Hinds, N. Chopra, T. Rantell, R. Andrews, V. Gavalas, and L. G. Bachas, Science 303, 62 (2004).

${ }^{10}$ K. Sint, B. Wang, and P. Kral, J. Am. Chem. Soc. 130, 16448 (2008).

${ }^{11}$ J. Dzubiella and J.-P. Hansen, J. Chem. Phys. 122, 234706 (2005).

${ }^{12}$ M. Majunder, X. Zhan, R. Andrews, and B. J. Hinds, Langmuir 23, 8624 (2007).

${ }^{13}$ J. Li, X. Gong, H. Lu, D. Li, F. Haiping, and R. Zhou, Proc. Natl. Acad. Sci. U.S.A. 104, 3687 (2007).

${ }^{14}$ B. Corry, J. Phys. Chem. B 112, 1427 (2008). 
${ }^{15}$ H. J. Hwang, K. R. Byum, and J. W. Kang, Physica E 23, 208 (2004).

${ }^{16}$ J. B. Freund, J. Chem. Phys. 116, 2193 (2002).

${ }^{17}$ A. Karla, S. Garde, and G. Hummer, Proc. Natl. Acad. Sci. U.S.A. 100, 10173 (2003).

${ }^{18}$ M. C. Gordillo and J. Martí, Chem. Phys. Lett. 329, 341 (2000).

${ }^{19}$ J. Rodriguez, M. D. Elola, and D. Laria, J. Phys. Chem. B 113, 12744 (2009).

${ }^{20}$ J. Rodriguez, M. D. Elola, and D. Laria, J. Phys. Chem. B 113, 7900 (2010).

${ }^{21}$ J. Rodriguez, M. D. Elola, and D. Laria, J. Phys. Chem. B 113, 14844 (2010).

${ }^{22}$ L. D. Gelb, K. E. Gubbins, R. Radhakrishnan, and M. SliwinskaBartkowiak, Rep. Prog. Phys. 62, 1573 (1999).

${ }^{23}$ L. D. Gelb and K. E. Gubbins, Phys. Rev. E 55, 1290 (1997).

${ }^{24}$ L. D. Gelb, M. Sliwinska-Barkwowiak, and K. Gubbins, in Fundamentals of Adsorption 6, edited by F. Maunier (Elsevier, Paris, 1998), p. 497.

${ }^{25}$ G. Rother, D. Woywod, M. Schoen, and G. H. Findenegg, J. Chem. Phys. 120, 11864 (2004)

${ }^{26}$ N. Giovambattista, P. J. Rossky, and P. G. Debenedetti, Phys. Rev. Lett. 102, 050603 (2009).

${ }^{27}$ M. Y. Lin, S. K. Sinha, J. M. Drake, X. I. Wu, P. Thiyagarajan, and H. B. Stanley, Phys. Rev. Lett. 72, 2207 (1994).

${ }^{28} \mathrm{~S}$. Kittaka, M. Kuranishi, S. Ishimaru, and O. Umahara, J. Chem. Phys. 126, 091103 (2007).

${ }^{29}$ J. Lum, D. Chandler, and J. D. Weeks, J. Phys. Chem. B 103, 4570 (1999).

${ }^{30}$ N. Giovambattista, P. J. Rossky, and P. G. Debendetti, Phys. Rev. E 73, 041604 (2006).

${ }^{31}$ D. Chandler, Nature (London) 437, 640 (2005).

${ }^{32}$ G. Hummer, J. C. Rasaiah, and J. P. Noworyta, Nature (London) 414, 188 (2001).

${ }^{33}$ A. Waghe, J. Rasaiah, and G. Hummer, J. Chem. Phys. 117, 10789 (2002).

${ }^{34}$ S. R. V. Castrillon, N. Giovambattista, I. A. Aksay, and P. G. Debenedetti, J. Phys. Chem. B 113, 1438 (2009).

${ }^{35}$ S. R. V. Castrillon, N. Giovambattista, I. A. Aksay, and P. G. Debenedetti, J. Phys. Chem. B 113, 7973 (2009).
${ }^{36}$ M. D. Elola, J. Rodriguez, and D. Laria, J. Chem. Phys. 133, 154707 (2010).

${ }^{37}$ J. Martí and M. C. Gordillo, Phys. Rev. B 63, 165430 (2001).

${ }^{38}$ P.-A. Cazade, J. Dweik, B. Coasne, F. Henn, and J. Palmeri, J. Phys. Chem. C 114, 12245 (2010).

${ }^{39}$ A. T. Beu, J. Chem. Phys. 132, 164513 (2010).

${ }^{40}$ L. Liu, X. Chen, W. Lu, A. Han, and Y. Qiao, Phys. Rev. Lett. 102, 184501 (2009).

${ }^{41}$ Y. W. Tang, K.-Y. Chan, and I. Szalai, J. Phys. Chem. B 108, 18204 (2004).

${ }^{42}$ R. M. Lynden-Bell and J. C. Rasaiah, J. Chem. Phys. 105, 9266 (1996).

${ }^{43}$ L. Yang and S. Garde, J. Chem. Phys. 126, 084706 (2007).

${ }^{44}$ W. Y. Lo, K. Y. Chan, M. Lee, and K. L. Mok, J. Electroanal. Chem. 450, 265 (1998)

${ }^{45}$ K. Shirono, N. Tatsumi, and H. Daiguji, J. Phys. Chem. B 113, 1041 (2009).

${ }^{46}$ C. D. Lorenz, P. S. Crozier, J. A. Anderson, and A. Travesset, J. Phys. Chem. C 112, 10222 (2008).

${ }^{47}$ M. C. F. Wander and K. L. Shuford, J. Phys. Chem. C 114, 20539 (2010).

${ }^{48}$ R. Zangi, M. Hage, and B. J. Berne, J. Am. Chem. Soc. 129, 4678 (2007).

${ }^{49}$ D. Argyris, D. R. Cole, and A. Striolo, ACS Nano 4, 2035 (2010).

${ }^{50}$ T. Kamijo, A. Yamaguchi, S. Suzuki, N. Teramae, T. Itho, and T. Ikeda, J. Phys. Chem. A 112, 11535 (2008).

${ }^{51}$ C. M. Morales and W. H. Thompson, J. Phys. Chem. A 113, 1922 (2009).

${ }^{52}$ T. S. Gulmen and W. H. Thompson, in Dynamics in Small Confining Systems VIII, Materials Research Society Symposium Proceedings 899E, edited by J. T. Fourkas, P. Levitz, R. Overney, and M. Urbakh (Materials Research Society, Warrendale, PA, 2005), 0988-N06-05.

${ }^{53}$ T. S. Gulmen and W. H. Thompson, Langmuir 25, 1103 (2009).

${ }^{54}$ J. C. Phillips, R. Braun, W. Wang, J. Gumbart, E. Tajkhorshid, E. Villa, C. Chipot, R. D. Skeel, L. Kale, and K. Schulten, J. Comput. Chem. 26, 1781 (2005).

${ }^{55}$ W. L. Jorgensen, J. Chandrashekhar, J. D. Madura, R. W. Impey, and M. L. Klein, J. Chem. Phys. 79, 926 (1983).

${ }^{56}$ J. Sala, E. Guàrdia, and J. Martí, J. Chem. Phys. 132, 215405 (2010).

${ }^{57}$ I.-C. Yeh and M. L. Berkowitz, J. Chem. Phys. 111, 3155 (1999).

${ }^{58}$ P. Jungwirth and D. J. Tobias, Chem. Rev. 106, 1259 (2006). 